\title{
Undersampling and the inference of coevolution in proteins
}

\author{
Yaakov Kleeorin ${ }^{1}$, William P. Russ ${ }^{2}$, Olivier Rivoire ${ }^{3 *}$, Rama Ranganathan ${ }^{1,4 *}$
}

\begin{abstract}
Protein structure, function, and evolution depend on local and collective epistatic interactions between amino acids. A powerful approach to defining these interactions is to construct models of couplings between amino acids that reproduce the empirical statistics (frequencies and correlations) observed in sequences comprising a protein family. The top couplings are then interpreted. Here, we show that as currently implemented, this inference is always biased, a problem that fundamentally arises from the distinct scales at which epistasis occurs in proteins in the context of limited sampling. We show that these issues explain the ability of current approaches to predict tertiary contacts between amino acids and the inability to obviously expose larger networks of functionally-relevant, collectively evolving residues called sectors. This work provides a necessary foundation for more deeply understanding and improving evolution-based models of proteins.
\end{abstract}

Keywords

coevolution - SCA - DCA - contact prediction - sector

${ }^{1}$ Center for Physics of Evolving Systems, Department of Biochemistry \& Molecular Biology, University of Chicago, Chicago, IL 60637

${ }^{2}$ Green Center for Systems Biology, University of Texas Southwestern Medical Center, Dallas, TX 75390

${ }^{3}$ Center for Interdisciplinary Research in Biology (CIRB), Collège de France, CNRS, INSERM, PSL Research University, F-75005 Paris, France

${ }^{4}$ The Pritzker School for Molecular Engineering, University of Chicago, Chicago, IL 60637

*Corresponding authors: olivier.rivoire@college-de-france.fr, ranganathanr@uchicago.edu

\section{Introduction}

The basic characteristics of natural proteins are the ability to fold into compact three-dimensional structures, to carry out chemical reactions, and to adapt as conditions of selection fluctuate. To understand how these properties are encoded in the amino acid sequence, a powerful approach is statistical inference from datasets of homologous sequences - the study of evolutionary constraints on and between amino acids. In different implementations, this approach has led to the successful prediction of protein tertiary structure contacts ${ }^{1-4}$, protein-protein interactions $^{5-7}$, mutational effects ${ }^{8-11}$, and even the design of synthetic proteins that fold and function in a manner indistinguishable from their natural counterparts ${ }^{12-14}$. A major result from these studies is the sufficiency of pairwise correlations in multiple sequence alignments to specify many key aspects of proteins. This result motivates the search for statistical models of protein sequences that capture these correlations as a route to understanding and designing proteins.

What characteristics underlie a "good" statistical model of protein sequences? The native state of a protein represents a fine balance of large opposing forces between atoms that operate with strong distance dependence to produce marginally stable structures. Thus, many complex and non-intuitive patterns of interdependence between amino acids (epistasis) are possible, all consistent with the compact, well-packed charac- ter of tertiary structures. Indeed, many studies show that amino acids act heterogeneously and cooperatively within proteins, producing epistasis between amino acids on vastly different scales. At one level, there are local, pairwise epistatic interactions that define direct contacts in the tertiary structure, which likely contribute to native-state stability. But, at another level, there are collectively acting networks of amino acids that mediate central aspects of protein function - binding ${ }^{15}$, cataly$\operatorname{sis}^{16}$, and allosteric communication ${ }^{17}$. Past work shows that both scales of couplings are represented in the pattern of empirical correlations in multiple sequence alignments (MSAs) ${ }^{18,19}$, providing different sequence-based methods for understanding protein structure ${ }^{20}$ and function $^{21}$. Thus, a basic requirement for statistical models of protein sequences is to account for both local and collective amino acid epistasis.

A fundamental problem in making such models is the lack of a ground truth for validating all features of the inference process. For example, the local epistasis can can be verified by direct contacts in atomic structures of members of a protein family ${ }^{1-4}$, but a similar benchmark for global collective actions of amino acids is not broadly available. Indeed the inspiration for building statistical models from evolutionary data is, in part, to provide hypotheses for the collective behaviors of amino acids as a route to understanding protein function. How then can we better understand the infer- 
bioRxiv preprint doi: https://doi.org/10.1101/2021.04.22.441025; this version posted April 23, 2021. The copyright holder for this preprint (which was not certified by peer review) is the author/funder, who has granted bioRxiv a license to display the preprint in perpetuity. It is made available under aCC-BY-NC-ND 4.0 International license.

Undersampling and the inference of coevolution in proteins $-2 / 12$

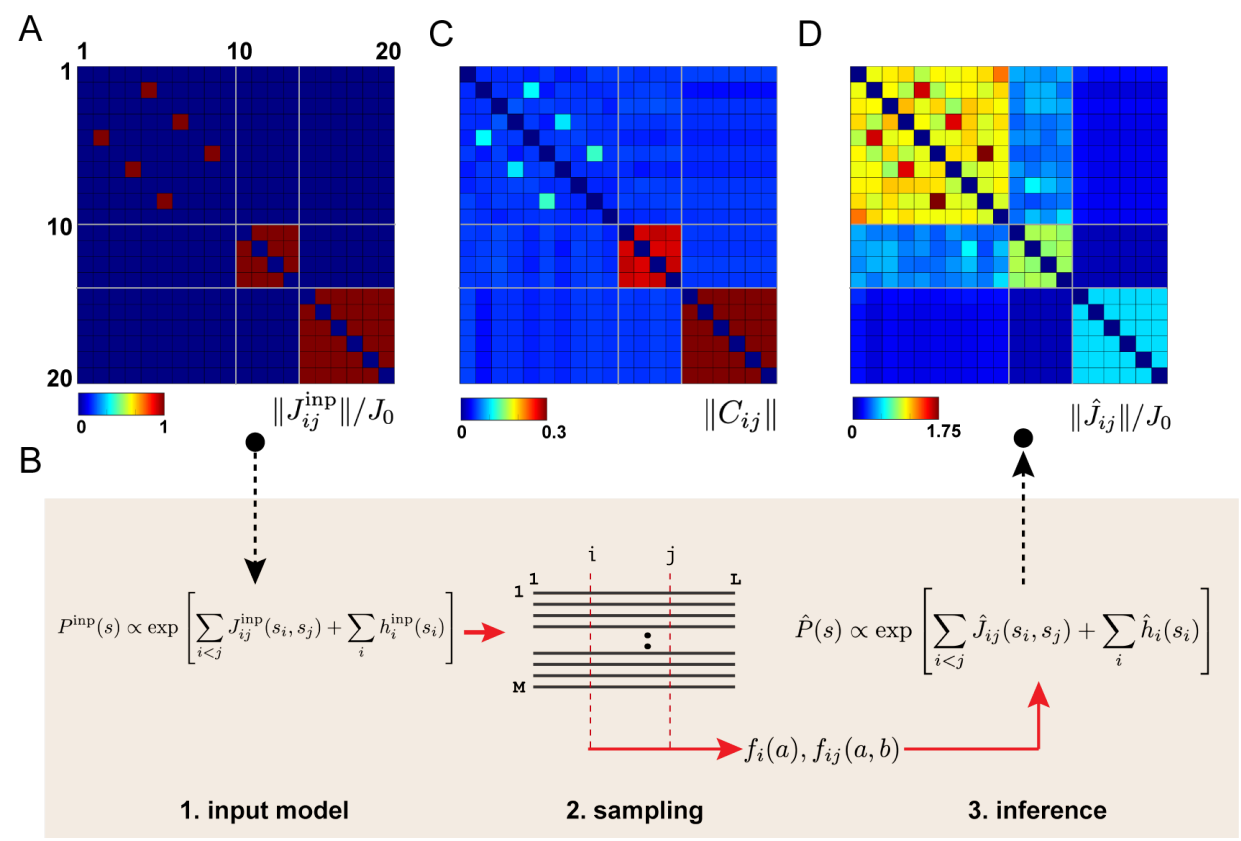

Figure 1. Inference from a toy model of proteins. The model assumes a sequence of length $L=20$ with $q=10$ possible amino acids at each position. A, the pattern of input couplings between sequence positions. There are three types of features: three isolated pairwise couplings ("contacts", 2-5, 4-7, and 6-9), a small collective group ("small sector", all possible couplings within positions 10-13), and a large collective unit ("large sector", all possible couplings within positions 14-20). All non-zero couplings have same magnitude, see text. B, the strategy used in this work, in which we make the input model (step 1), sample $M$ sequences from a Boltzmann distribution defined by the input $J_{i j}^{\text {inp }}(a, b)$ and compute the empirical first and second order statistics $f_{i}(a)$ and $f_{i j}(a, b)$ (step 2), and use the DCA approach to infer back the input couplings from the sampled sequences (step 3). C, Frobenius norm $\left\|C_{i j}\right\|$ of the empirical correlation matrix $C_{i j}(a, b)=f_{i j}(a, b)-f_{i}(a) f_{j}(b)$ computed from the sampled sequences, showing that the collective groups are most strongly correlated. $\mathbf{D}$, the inferred couplings with usual settings in DCA (regularization $\lambda_{J}=10^{-3}$ ). As described in the text, panels $\mathbf{A}$ and $\mathbf{D}$ show normalized couplings $\left|\hat{J}_{i j}\right| / / J_{0}$.

ence process itself? In this work, we take the approach of using "toy models" $22-24$ in which we (1) specify a pattern of amino acid couplings for a hypothetical protein, (2) generate synthetic sequences that satisfy those constraints, and (3) examine the ability of statistical inference methods to learn these patterns (Fig. 1A-B). This analysis shows that in any practical context, model inference is biased by the limited sampling of sequences. The consequence is that features of different size and strength are unevenly inferred with current methods. These findings are confirmed in a real protein model system in which experimental data allow us to verify both structural contacts and functional amino acid networks. This work clarifies apparent inconsistencies in the current interpretation of coevolution in proteins and opens a path towards new methods for more completely inferring the information content of protein sequences.

\section{Results}

\section{Inference from toy models}

A generative statistical model of protein sequences is provided by the Direct Coupling Analysis (DCA) ${ }^{3,20}$, or more generally a Markov random field. This method starts with a multiple sequence alignment (MSA) of a protein family comprised of $M$ sequences by $L$ positions, and makes the assumption that each sequence $s=\left(s_{1}, \ldots, s_{L}\right)$ is a sample from a Boltzmann distribution of a Potts model,

$$
P(s) \propto \exp \left[\sum_{i} h_{i}\left(s_{i}\right)+\sum_{i<j} J_{i j}\left(s_{i}, s_{j}\right)\right]
$$

where $h_{i}(a)$ represents the intrinsic propensity of each amino acid $a$ to occur at each position $i$ (the "fields"), $J_{i j}(a, b)$ represents the constraints between amino acids $a, b$ at pairs of positions $i, j$ (the "couplings"), and $P(s)$ is the probability of sequence $s$.

The parameters $(h, J)$ are inferred by maximum likelihood and are related to the frequencies $f_{i}(a)$ and joint frequencies $f_{i j}(a, b)$ of amino acids at positions $i, j$ by the consistency equations

$$
\begin{aligned}
f_{i}(a) & =\sum_{s} P(s) \delta\left(s_{i}, a\right) \\
f_{i j}(a, b) & =\sum_{s} P(s) \delta\left(s_{i}, a\right) \delta\left(s_{j}, b\right)
\end{aligned}
$$

where $\delta(x, y)=1$ when $x=y$ and zero otherwise. The probability distribution $P(s)$ can also be viewed as the maximum entropy model that reproduces the empirical frequencies $f_{i}(a)$ and $f_{i j}(a, b)$ of the protein family ${ }^{3}$. 
In practice, exact inference of the parameters $(h, J)$ is computationally intractable because of the number of terms in Eq. (2) is excessively large, but effective approximations exist. In this work, we use pseudo-likelihood maximization (plmDCA) ${ }^{25}$, but the results are consistent with other approximations, including Boltzmann machine learning (bmDCA $)^{26}$. When possible, we make use of exact calculations (Fig. S1).

A critical fact is that in any practical situation, the inference is carried out in the limit of extremely poor sampling. Typically, MSAs contain on the order of $10^{3}-10^{4}$ effective sequences while the number of parameters $(h, J)$ to be estimated is on the order of $10^{5}-10^{7}$. This undersampling necessitates the use of statistical regularization during the inference process to avoid overfitting. A standard approach is the so-called $L_{2}$ regularization, meaning that the log-likelihood function is penalized by a term proportional to the $L_{2}$ norm of the parameters. The larger the regularization, the more constrained the parameters. If the fields $h_{i}(a)$ and the couplings $J_{i j}(a, b)$ are regularized separately, this changes the consistency equations to

$$
\begin{aligned}
f_{i}(a) & =\sum_{s} P(s) \delta\left(s_{i}, a\right)+\lambda_{h} h_{i}(a) \\
f_{i j}(a, b) & =\sum_{s} P(s) \delta\left(s_{i}, a\right) \delta\left(s_{j}, b\right)+\lambda_{J} J_{i j}(a, b)
\end{aligned}
$$

where $\lambda_{h}$ and $\lambda_{J}$ are the regularization parameters. How does one choose these parameters? Since the inference is unsupervised and cross-validation strategies cannot be applied, the standard approach is to empirically set them by predictive power for various protein properties of interest ${ }^{9,25}$.

To more formally study the influence of sample size and regularization on the inference process, we made a toy model of a hypothetical protein obeying Eq. (1) with input parameters $\left(h^{\mathrm{inp}}, J^{\mathrm{inp}}\right)$, and asked whether these parameters can in fact be inferred from sequences sampled from the model (Fig. 1B). The model comprises $L=20$ positions and $q=10$ possible amino acids and has the following characteristics: all fields are set to zero $\left(h_{i}^{\text {inp }}(a)=0\right)$, and couplings $J_{i j}^{\text {inp }}(a, b)$ have the pattern shown in Fig. 1A. There are three isolated pairwise couplings at pairs of positions $(2,5),(4,7)$, and $(6,9)$, a medium-sized interconnected group containing all possible couplings between positions (10-13), and a larger-sized interconnected group containing all possible couplings within positions (14-20). The isolated pairwise couplings mirror the concept of coevolving contacts in protein structures while the interconnected groups of couplings represent the concept of a cooperatively evolving group of positions (sectors). All non-zero couplings have the same strength $J^{\text {inp }}=2$. Note that $J_{i j}(a, b)$ is a four-dimensional $L \times L \times q \times q$ array, but for presentation, Fig. 1A (and all such panels below) shows the $L \times L$ Frobenius norm $\left\|J_{i j}\right\|=\left(\sum_{a, b} J_{i j}(a, b)^{2}\right)^{1 / 2}$ (see methods). We also normalize inferred parameters by the input value, so that perfect inference corresponds to $\left\|\hat{J}_{i j}\right\| / J_{0}=1$ for all non-zero couplings.

We used a Markov Chain Monte Carlo sampling procedure to draw an MSA of $N=300$ independent sequences from the model (Fig. 1B, step 2), a number that mirrors the undersampling observed in natural protein families. Fig. $1 \mathrm{C}$ shows the position by position magnitudes of correlations between amino acids in the sampled sequences. The pattern is heterogeneous, with stronger correlations within the larger interconnected groups of positions. This is because the larger the group, the less likely a position within it is to change over the sampled sequences. In this context, how does DCA work to infer the input couplings $J_{i j}(a, b)$ from the empirical statistics? With standard settings for regularization $\left(\lambda_{J}=10^{-3}\right)$, DCA emphasizes the isolated pairwise couplings while the collective features are hardly discernable relative to noise (Fig. 1D).

\section{Inference as a function of sample size}

Why does DCA selectively emphasize the isolated couplings and under-represent those that make up larger collective features? The answer lies in examining the dependence of the inferred couplings $\hat{J}_{i j}(a, b)$ on the degree of sampling in the MSA (Fig. 2A). The data indicate that inferred couplings show three properties as a function of MSA size: (1) they exhibit a "resonance" property where the value of $\left\|\hat{J}_{i j}\right\|$ sharply peaks at a characteristic MSA size, (2) they resonate at different characteristic MSA sizes depending on the size of the group they belong to (isolated pairs, small collective, and large collective units), and (3) they only approach their correct values $\left(\left\|\hat{J_{i j}}\right\| / J_{0}=1\right)$ at the limit of very large sampling. At the MSA size chosen in our example $(N=300)$, the isolated couplings dominate the inference, with all collective features many-fold lower in magnitude. However, Fig. 2A also shows that if the MSA contained increasingly more sequences, the outcome would be different; we would suppress the isolated pairwise couplings and instead emphasize the collective features (see Fig. S2 for more details).

What is the mechanism of the resonance of inferred couplings as a function of MSA size? To study this, we made an even simpler model of just two positions, each with $q$ possible amino acids and with no fields or couplings; that is, with no constraints at all. With infinite sampling, all correlations between amino acids at the two positions must be zero and the inference will return the correct result that all fields and couplings are zero. With finite number of sequences, however, the inferred parameters $(\hat{h}, \hat{J})$ are generally non-zero. For example, consider the situation in which we deterministically draw amino acid pairs uniquely and with- 
bioRxiv preprint doi: https://doi.org/10.1101/2021.04.22.441025; this version posted April 23, 2021. The copyright holder for this preprint (which was not certified by peer review) is the author/funder, who has granted bioRxiv a license to display the preprint in perpetuity. It is made available under aCC-BY-NC-ND 4.0 International license.

Undersampling and the inference of coevolution in proteins $-4 / 12$
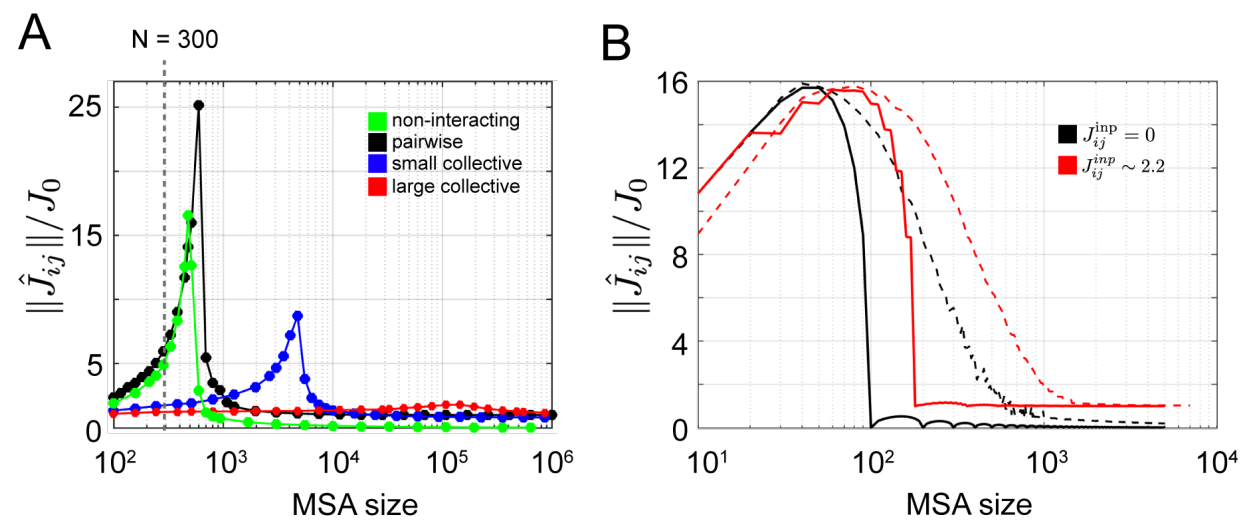

Figure 2. Resonance in inference of sequence features. A, normalized magnitude of inferred couplings $\left\|\hat{J}_{i j}\right\| / J_{0}$ as a function of MSA size, averaged for positions comprising the diffrent sized features in the input model (Fig. 1A). The inference is carried out with weak regularization $\left(\lambda_{I}=10^{-6}\right)$. The data show that features in the amino acid sequence display a resonance at characteristic levels of sampling in order of their effective size. Interactions of any size only reach their true values $\left(\left\|\hat{J}_{i j}\right\| / J_{0}=1\right)$ at the limit of infinite sampling. B, Inferred couplings for an even simpler model of just $L=2$ positions and $q=10$ amino acids either without (black, $J^{\text {inp }}=0$ ) or with $J^{\text {inp }} \simeq 2.2$ (red) input interactions. The traces show cases of deterministic (solid) or random (dashed) sampling of sequences. As described in the text, this model provides a simple mechanistic understanding of the origin of the resonance property.

out repetition to form an MSA of size $N$ while keeping amino acid frequencies at both sites uniform. If $N<q^{2}$, some amino acid pairs will be observed and the rest $\left(q^{2}-N\right)$ will be absent, requiring inferred couplings in the Potts model to be infinite to account for the absences. The point of regularization is to prevent such an outcome, constraining the difference between the largest and smallest couplings $(\Delta J)$ to satisfy

$$
\Delta J+\log \Delta J=\log \frac{q^{2}-N}{N^{2} \lambda_{J}},
$$

where $\lambda_{J}$ is the regularization parameter. It is then easy to show that the magnitude of couplings over all amino acid pairs will be unimodal, with a maximum at the point where the sampling produces the same number of observed and missing pairs (that is, when $N=q^{2} / 2$ ) (Fig. 2B, solid black curve, and see SI for derivation). The true value of the interaction $(J=0)$ is only reached with complete sampling $\left(N \gg q^{2}\right)$. This shows the basic mechanism of resonance - a sampling-dependent maximization of inferred couplings with a magnitude that is simply set by the strength of regularization (Eq. (4)).

Generalizing to include a non-zero input coupling (red curve in Fig. 2B) has the effect of displacing the resonance curve to the right and shifting the inferred coupling at large sampling to the correct input value (Fig. 2B, compare black and red curves). This makes sense; with stronger coupling, more sampling is generally necessary to draw all possible amino acid states. Thus, as shown in Fig. 2A, the position of a resonance peak is a function of the effective size and magnitude of the input interaction. Pure sampling noise in uncoupled positions resonates at the lowest MSA size, followed in sequence by isolated pairwise couplings and collective features of increasing size. Relaxing the model to use random, rather than deterministic sampling of amino acid pairs just further increases the sampling required for inferring couplings, either without (Fig. 2B, black dashed curve) or with (Fig. 2B, red dashed curve) true interactions.

The implications for sequence-based DCA models of real proteins are clear. Inference for any protein family always occurs at the limit of extreme poor sampling, below the resonance peak for isolated pairwise couplings. In this regime, with usual small regularization to suppress sampling noise, couplings inferred are dominated by the smallest scale features of the information stored in amino acid sequences. Thus the inferred couplings emphasize isolated pairwise couplings between positions that correspond to structural contacts, with couplings within collective groups at or even below the level of pure sampling noise.

The toy model provides another insight into the contact prediction process. A common practice in DCA is to apply an Average Product Correction (APC), which removes a background value from inferred couplings ${ }^{27}$. This approach has been justified by its role in mitigating the effects of phylogenetic bias. However, APC also improves the inference of isolated contacts in our toy model, which includes no notion of phylogeny (Fig. S3). The work here suggests a more general explanation for APC: it works by suppressing the spurious couplings between non-interacting positions that arise due to extreme undersampling. Since in this limit, the noninteracting couplings are comparable in magnitude to the smallest scale of true couplings between positions (Fig. 2), APC helps to separate signal from noise and improve contact prediction in protein structures (Fig. 
bioRxiv preprint doi: https://doi.org/10.1101/2021.04.22.441025; this version posted April 23, 2021. The copyright holder for this preprint (which was not certified by peer review) is the author/funder, who has granted bioRxiv a license to display the preprint in perpetuity. It is made available under aCC-BY-NC-ND 4.0 International license.

Undersampling and the inference of coevolution in proteins $-5 / 12$
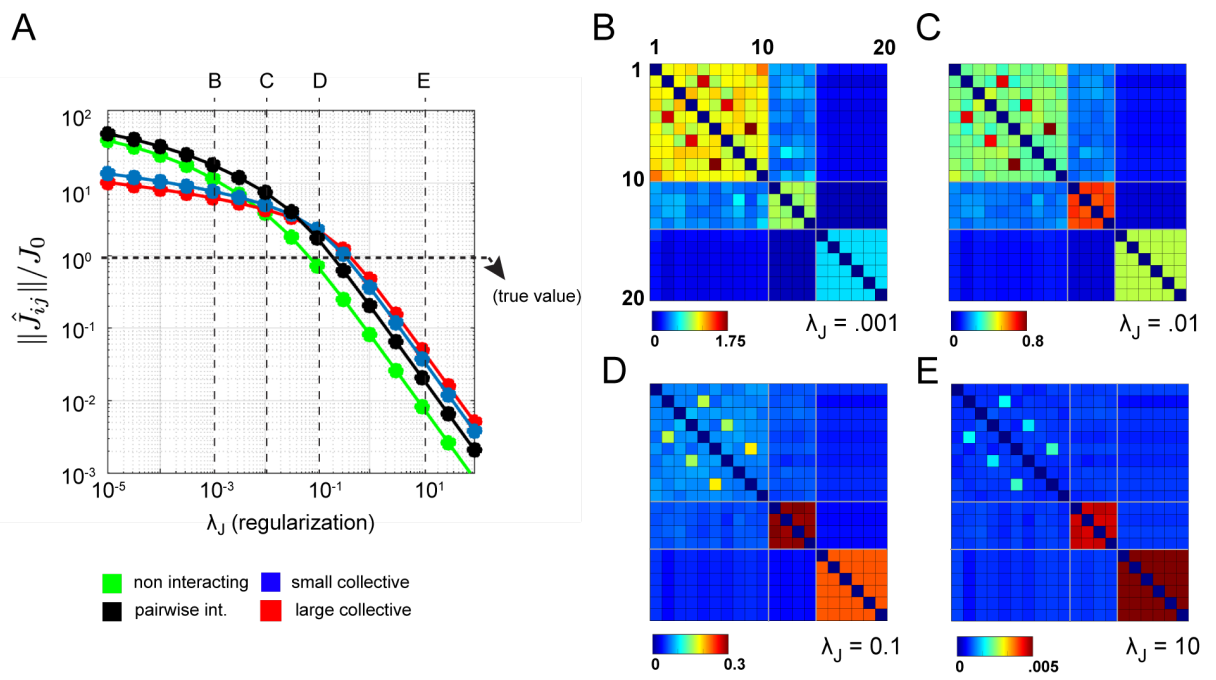

Figure 3. Inference of couplings as a function of the regularization parameter $\lambda_{J}$. A, the normalized magnitude of inferred couplings $\left\|\hat{J}_{i j}\right\| / J_{0}$ averaged over position pairs comprising isolated pairwise couplings (black), for the small sized collective group (blue), and for the large-sized collective group (red). Inferred couplings for position pairs with zero input couplings are pure undersampling noise and are shown in green. Values of $\lambda_{J}$ corresponding to panels B-E are marked, and the true value for non-zero couplings is indicated. B-E, for comparison with Fig. 1A, the $\hat{J}_{i j}$ matrix inferred at increasing levels of regularization $\lambda_{J}$. The data show how features of different effective size dominate the inference as regularization is adjusted from small to large values. Note that DCA is traditionally carried out at small regularization strengths $\lambda_{J}<10^{-2}$.

S3).

\section{Inference as a function of regularization strength}

As explained above, the magnitude of inferred couplings in the undersampled regime is basically set by the strength of the regularization parameter $\lambda_{J}$ (Eq. (4)). For example, with typical small $\lambda_{J}, \Delta J \sim-\log \lambda_{J}$. But how do features of different sizes respond to regularization? To understand this, we carried out model inference for a fixed size MSA $(N=300)$ drawn from the toy model while varying the regularization strength $\lambda_{J}$ (Fig. 3A). The data show that for small regularization, the isolated pairwise couplings dominate (black), and collective features (blue and red) are inferred at or below the level of non-interacting pairs (green). As regularization is increased, different features take prominence, until ultimately features are inferred with magnitudes that are in order of their effective size - large collective $>$ small collective $>$ isolated pairs (Fig. 3A). In this strong regularization regime, all inferred couplings decay like $1 / \lambda_{J}$ and resemble the empirical correlations $C_{i j}(a, b)$ (see SI for details). Remembering that the true input couplings are equal for all features and have normalized value $\left\|J_{i j}^{\text {inp }}\right\| / J_{0}=1$, we can conclude that there is no single choice of a regularization parameter that can correctly infer the true pattern of couplings whenever sampling of sequences is limited (compare Fig. 3B-E with Fig. 1A).

An even simpler model with just two features and two parameters provides an intuitive geometrical illustration of the problem (Fig. 4). This model comprises sequences with $L=6$ positions and $q=2$ amino acids
A

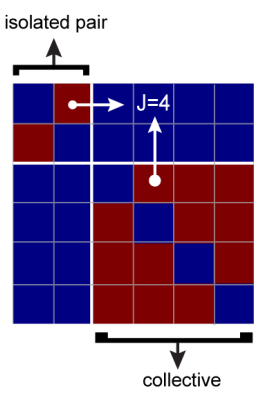

B

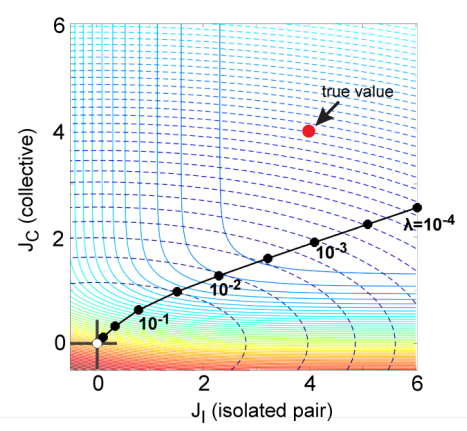

Figure 4. A geometrical explanation of regularized inference. A, the input coupling matrix $J^{\text {inp }}$ for a toy model with $L=6$ positions and $q=2$ amino acids and with no fields $h$. The model has two parameters, one representing the isolated pairwise coupling $\left(J_{I}\right.$, positions 1-2) and the other the couplings in the collective set $\left(J_{S}\right.$, positions 3-6). The input values are $J_{I}^{\text {inp }}=J_{C}^{\text {inp }}=4$. B, Inferred values of $J_{I}$ and $J_{C}$ from an $N=4$ undersampled set of sequences for the toy model as a function of regularization $\lambda_{J}$. The solid contours show the landscape of the log-likelihood function being optimized, and the dashed contours show values of $\left(\hat{J}_{I}, \hat{J}_{C}\right)$ that are consistent with different strengths of regularization.

with a pattern of input interactions $J^{\text {inp }}$ shown in Fig. 4A. There is one isolated pairwise coupling between positions 1 and $2\left(J_{I}\right)$, and one collective group of couplings between positions 3-6 $\left(J_{C}\right)($ Fig. $4 \mathrm{~A})$, all with the same magnitude $J_{I}^{\text {inp }}=J_{C}^{\text {inp }}=4$. The value of the coupling is chosen simply to be significantly above random fluctuations. This makes the number of parameters to be inferred just two - $\left(J_{I}, J_{C}\right)$ - enabling us to visual- 
bioRxiv preprint doi: https://doi.org/10.1101/2021.04.22.441025; this version posted April 23, 2021. The copyright holder for this preprint (which was not certified by peer review) is the author/funder, who has granted bioRxiv a license to display the preprint in perpetuity. It is made available under aCC-BY-NC-ND 4.0 International license.

Undersampling and the inference of coevolution in proteins $-6 / 12$

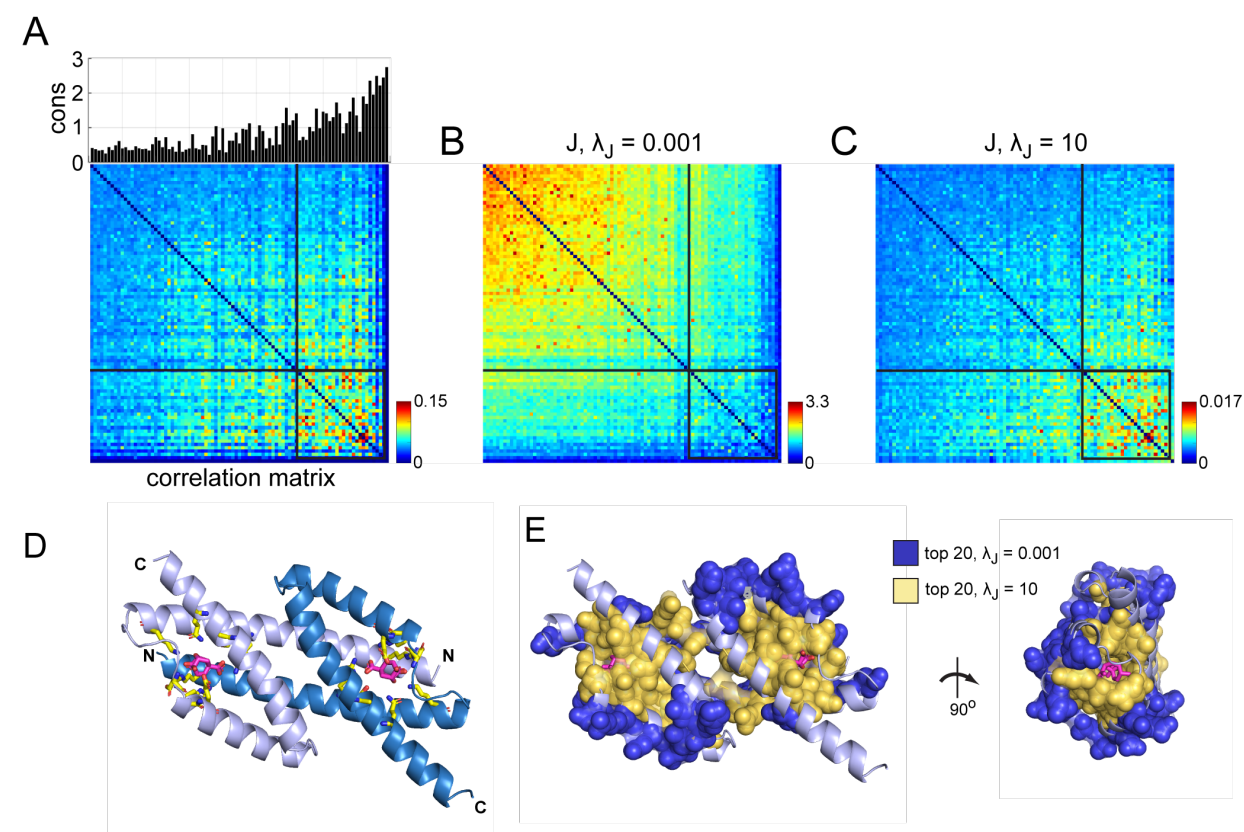

Figure 5. Inference of positional couplings for the AroQ family of chorismate mutase (CM) enzymes. A, positional conservation (by Kullback-Leibler relative entropy ${ }^{21}$, bar graph) and the matrix of positional correlations for an MSA of 1258 CM homologs. The positions are ordered by "susceptibility to regularization" (see text and Supplementary Information). B-C, the coupling $\hat{J}_{i j}$ matrix for the CM family inferred with standard small regularization $\left(\lambda_{J}=0.001\right.$, panel B) or strong regularization $\left(\lambda_{J}=10\right.$, panel C), both ordered as in panel A. D, AroQ CMs are dimers with two symmetric active sites formed by elements from both protomers (blue and silver); active site residues are highlighted in yellow stick bonds and a bound substrate analog in magenta. Shown is the structure of the E. coli CM domain (EcCM, PDB 1ECM). E, spatial organization of positions comprising the top 20 couplings inferred with weak $\left(\lambda_{J}=0.001\right.$, blue spheres) or strong $\left(\lambda_{J}=10\right.$, orange spheres) regularization.

ize the inference results on a $2 \mathrm{D}$ plane (Fig. 4B). For an undersampled case (here, $N=4$ ), the contours of the log-likelihood function being optimized (solid blue contours) show that the inference process has no finite maximum; without regularization, inferred values of couplings $J_{I}, J_{C}$ will diverge to infinity. This is consistent with the intuition that couplings must be infinity to account for unobserved amino acid configurations.

How does regularization correct this problem? The dashed line contours in Fig. 4B show the curves along which the magnitude of $J_{i j}$ (that is, $J_{I}^{2}+6 J_{C}^{2}$ ) is a constant for various regularization strengths. This defines the solutions to inference with regularization - the points (black filled circles, Fig. 4B) where the solid contours are tangent to the dashed contours. Thus, the inferred solution is set by the regularization used, and there is no regularization at which the inferred solution matches the true solution $\left(J_{I}=J_{C}=4\right)$. Also, note that at this level of undersampling, $J_{I}$ is always larger than $J_{C}$. An analytical solution relating the regularization parameter $\lambda_{J}$ and inferred values of $\left(\hat{J}_{I}, \hat{J}_{C}\right)$ shows how the ratio of these parameters depends on the relative size of the pairwise and collective units, and on the level of sampling (see Supplementary Information).

The bottom line is that with undersampling of data, uniform regularization will always produce solutions that unequally represent the contribution of different sized features and more importantly, that will deviate from the ground truth.

\section{Application to real problems}

These findings have significant impact for model inference in real proteins. The pattern of empirical correlations between pairs of positions in MSAs of protein families reveals a hierarchy of correlation scales, both in terms of magnitude and size of the correlated unit. For example, in an MSA of 1258 members of the AroQ family of chorismate mutase enzymes, a subset of more conserved positions display a pattern of strong interconnected correlations and the remainder of less conserved positions show weaker and more dispersed correlations ${ }^{14}$ (Fig. 5A). This pattern is reminiscent of Fig. 1C, the correlation matrix resulting from a toy model with features of different effective size. Positions in Fig. 5A are ordered by their susceptibility to regularization (see methods), suggesting that with the extreme undersampling that characterizes all practical MSAs, the inference of couplings in Potts models will inevitably treat these groups unequally. Indeed, just like for the toy model, $\hat{J}_{i j}$ for the AroQ family inferred with standard weak regularization $\left(\lambda_{J}=0.001\right.$, Fig. $\left.5 \mathrm{~B}\right)$ highlights interactions between the weakly conserved positions while 
bioRxiv preprint doi: https://doi.org/10.1101/2021.04.22.441025; this version posted April 23, 2021. The copyright holder for this preprint (which was not certified by peer review) is the author/funder, who has granted bioRxiv a license to display the preprint in perpetuity. It is made available under aCC-BY-NC-ND 4.0 International license.

Undersampling and the inference of coevolution in proteins $-7 / 12$

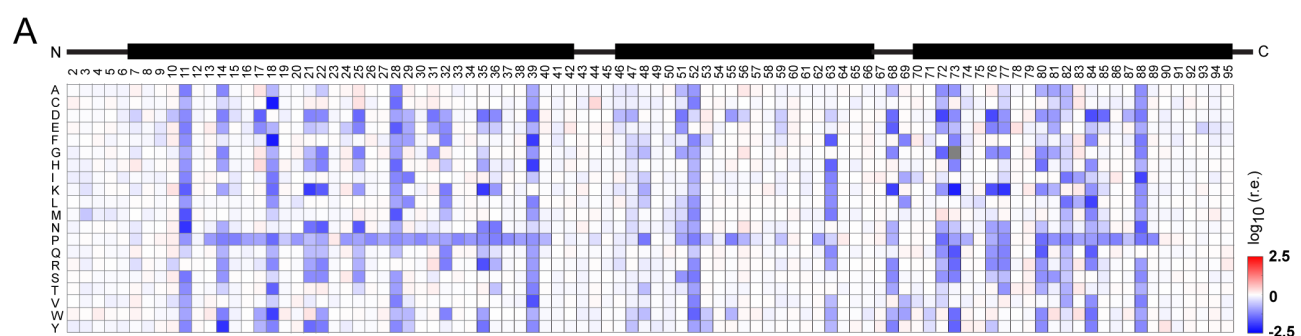

B

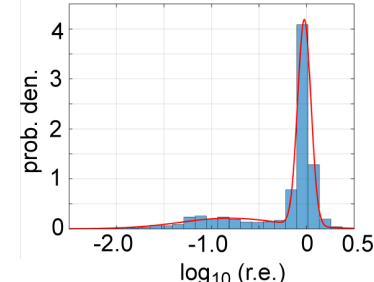

C

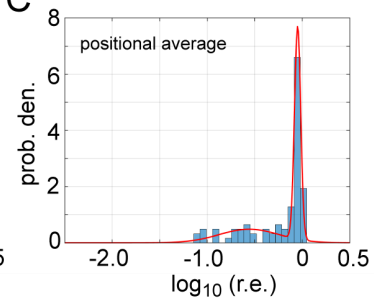

E

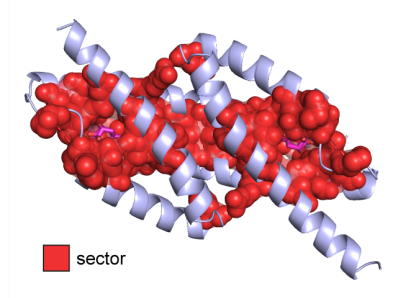

D

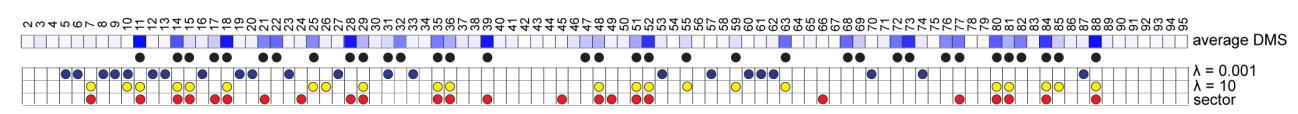

Figure 6. Functional analysis of positions in the E. coli CM domain. A, A deep mutational scan (DMS), showing the effect of every single mutation on the catalytic power relative to wild-type (see Methods). Blue shades indicate loss-of-function, red indicates gain-of-function, and white is neutral. The cartoon above indicates the secondary structure. B-C, the distribution of mutational effects displayed for all amino acid substitutions (B) or for the average effect of mutations at each position (C). The data are fit to a Gaussian mixture model with two components (red curve). D, the average effect of mutations is shown as a heatmap and circles below mark the positions comprising the deleterious mode in panel C (black), positions comprising the top 20 couplings inferred with weak $\left(\lambda_{I}=10^{-3}\right.$, blue) or strong $\left(\lambda_{I}=10\right.$, yellow) regularization, and positions comprising the sector as defined by the SCA method (red). E, the sector forms a physically contiguous network within the core of the $\mathrm{CM}$ enzyme linking the two active sites across the dimer interface.

inference with strong regularization $\left(\lambda_{J}=10\right.$, Fig. $\left.5 \mathrm{C}\right)$ highlights interactions between the conserved, more collectively evolving positions (Fig. S4). Thus, inference in the context of undersampling selectively represents the information content of protein sequences, with the magnitude of inferred couplings set by the regularization used (see color scale, Fig. 5B-C).

How do these findings influence our understanding of protein structure and function? AroQ CMs occur in bacteria, archaea, plants, and fungi and catalyze the conversion of the intermediary metabolite chorismate to prephenate, a reaction essential for biosynthesis of the aromatic amino acids tyrosine and phenylalanine. Structurally, these enzymes form a compact domain-swapped dimer of relatively small protomers with two active sites (Fig. 5D). The top terms in $\hat{J}_{i j}$ inferred with weak regularization $\left(\lambda_{J}=0.001\right)$ correspond to direct contacts between amino acids in the three-dimensional structure (Fig.S5), but are exclusively located within surfaceexposed residues (Fig. 5E, blue spheres). In contrast, top couplings inferred with strong regularization $\left(\lambda_{J}=10\right)$ switch to represent interactions between buried positions built around the enzyme active site (Fig. 5E, orange spheres). The couplings inferred with strong regularization include direct tertiary structure contacts, but also comprise indirect, longer-range or substrate-mediated interactions (Fig.S6). The key result is that regulariza- tion gradually shifts the pattern of inferred couplings from direct contacts at surface sites to a mixture of direct and indirect interactions within the protein core.

What is the functional meaning of these findings? To comprehensively evaluate this, we carried out a saturation single mutation screen (a "deep mutational scan") of the AroQ CM domain from E. coli (EcCM), following the effect on catalytic activity. This work is enabled by a quantitative select-seq assay for CM activity, reported recently ${ }^{14}$. Briefly, a library comprising all single mutations was made by oligonucleotide-directed NNScodon mutagenesis, expressed in a CM-deficient E. coli host strain (KA12/ pKIMP-UAUC, see Methods), and grown together as a single population under selective conditions. Deep sequencing of the populations before and after selection provides a log relative enrichment score for each mutant relative to wild-type which quantitatively reports the effect on catalytic power ${ }^{14}$. This information is displayed as a heatmap in Fig. 6A - a global survey of mutational effects in EcCM.

The distribution of mutational effects is bimodal (Fig. 6B-C), with one mode representing neutral variation and the other representing deleterious effects (black circles, Fig. 6D). The comparison with positions inferred in the top couplings of $\hat{J}_{i j}$ is clear - the top couplings inferred with standard weak regularization occur almost exclusively at mutationally tolerant positions, while 
bioRxiv preprint doi: https://doi.org/10.1101/2021.04.22.441025; this version posted April 23, 2021. The copyright holder for this preprint (which was not certified by peer review) is the author/funder, who has granted bioRxiv a license to display the preprint in perpetuity. It is made available under aCC-BY-NC-ND 4.0 International license.

Undersampling and the inference of coevolution in proteins $-8 / 12$

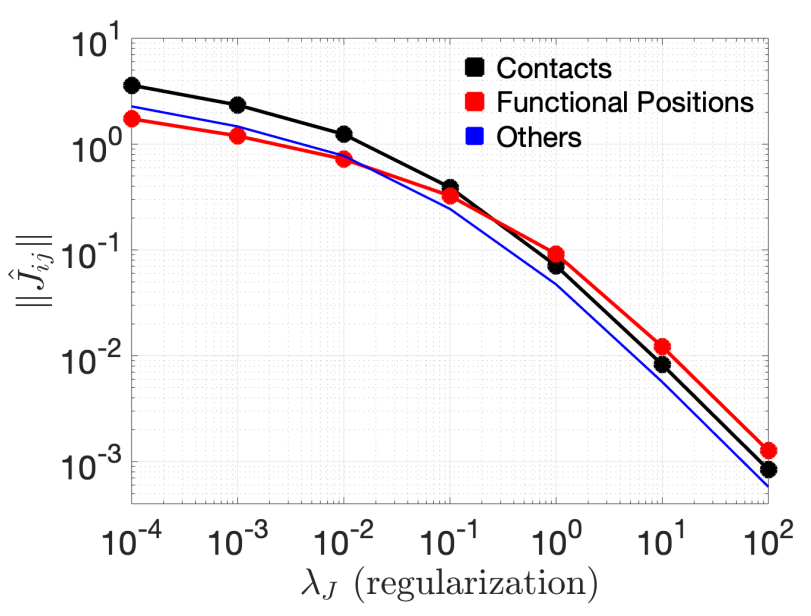

Figure 7. Inferred couplings as a function of regularization $\lambda_{J}$ in the $\mathrm{CM}$ protein family. The graph shows the magnitude of inferred couplings $\hat{J}_{i j}(a, b)$ averaged over significant couplings in functional positions as defined in Fig. 6 (red), direct contacts (black), and all other position pairs (light blue). Positions comprising three groups are given in the Suppleentary Information. In analogy with inference for toy models (Fig. 3A), these data show that features of different effective size (here, isolated pairwise contacts and interactions in a collective network) differentially dominate the inference as regularization is adjusted from small to large values.

those inferred with strong regularization occur at functionally important positions (Fig. $6 \mathrm{D}, p=3 \times 10^{-8}$, Fisher Exact Test) (Fig. S7-S8). Consistent with this, the top couplings inferred with strong regularization significantly overlap with the network of conserved, epistatically-coupled, functionally-relevant positions (the sector) defined by the statistical coupling analysis (SCA) method $^{21}$ ( $p=1.9 \times 10^{-7}$, Fisher Exact Test) (Fig. 6D-E).

A systematic analysis of the effect of regularization on inference of positional couplings is shown in Fig. 7. The data show that contacts and functional positions are differentially emphasized, with contacts acting like isolated pairwise couplings and functional sites acting like a more epistatic collective unit.

\section{Conclusion}

The inference of coevolution between amino acids has been valuable, providing new hypotheses for protein mechanisms and global rules for design. One approach is based on Potts models, in which empirical frequencies and correlations of amino acids in a multiple sequence alignment are used to define a probability distribution for the protein family over all sequences ${ }^{20}$. The Potts model has been demonstrated to reveal pairwise tertiary contacts between amino acids ${ }^{3,20}$, opening the path to sequence-based structure prediction ${ }^{1,4}$. In this regard, the apparent inability of Potts models to obviously describe collective interactions of amino acids has been puzzling ${ }^{28}$. The collective interactions have been shown to specify native-state foldability ${ }^{13}$, biochemical activities $^{10,12,15,16,29,30}$, allosteric communication ${ }^{17,31,32}$, and evolvability ${ }^{33}$, defining features of proteins that are essential for their biological function.

The work presented here explains the nature of this problem. With limited sampling of sequences in practically available MSAs, features of different effective size and conservation will be differentially emphasized as a function of MSA size and regularization. With weak regularization, the inference will focus on small-scale, relatively unconserved, functionally-less important local structural interactions, and with strong regularization, inference will emphasize larger-scale, conserved, functionally-essential features. A key point is that there is no single setting of regularization at which all features will be correctly represented. We illustrate this concept using Potts models and one form of regularization $\left(L_{2}\right)$, but the principle of heterogeneous inference of features of different scale is a general one, and depends only on working in the undersampled regime.

The consequence of biased inference is clearly seen in the use of Potts models for protein design. Recent work shows that sequences drawn from a Potts models of chorismate mutate enzymes are indeed true synthetic homologs of the protein family, displaying function both in vitro and in vivo that recapitulates the activity of the natural counterparts ${ }^{14}$. However, this result required sampling from the model at computational temperatures less than unity, a process that is meant to shift the energy scale to correct for regularization and to enforce under-estimated but functionally essential couplings. This procedure nicely recovers protein function, but does so at the expense of dramatic reduction in sequence diversity of designed proteins compared to natural ones ${ }^{14}$. In light of the work presented here, we can now understand this problem as a non-optimal solution to compensating the unequal inference of features by globally depressing the energy scale.

Can we then "correct" the inference process to more uniformly and accurately represent the biologically relevant patterns of amino acid interactions? Given that practical MSAs will always be grossly undersampled, the main parameter we can control is regularization. But since no single regularization parameter can provide a proper inference of all scales of interactions, it seems clear that what is needed is a strategy for inhomogeneous regularization, where parameters in the Potts model are inferred according to the level of sampling noise that acts on them. If done correctly, such a process should lead to a model that unifies the inference of both local and collective features and enables design of artificial proteins that recapitulate the sequence diversity of natural members of a protein family. The computational toy models introduced here, and the availability of powerful experimental systems such as the choris- 
mate mutases may provide the foundation for this next advancement in sequence based models for proteins.

\section{Methods}

\section{Toy models and simulated data}

Simulated data are generated from input Potts models, with couplings and fields $\left\{J^{\text {inp }}, h^{\text {inp }}\right\}$. The inferred couplings and fields are denoted $\{\hat{J}, \hat{h}\}$. The input model described in Figs. 1-3 involves zero fields $\left(h_{i}^{\text {inp }}(a)=0\right)$ and couplings with non-zero interactions set to equal strength $\left(J_{i j}^{\mathrm{inp}}(a, a)=2\right)$. This choice makes the pattern of couplings favorable for $i$ and $j$ to have identical amino acids, excluding frustration. Sequences are generated from input models through a Markov-Chain Monte Carlo process using the Metropolis-Hastings algorithm. Each sample is obtained after $2 \cdot 10^{5}$ Monte Carlo iterations starting from independent random sequences. All codes were written in house using MATLAB (Mathworks Inc.) and are available upon request.

\section{Inference and Gauge}

Exact calculations were used for model inference in the small systems described in Figs. 2B and 4. The process involves numerical minimization of the negative $\log$ likelihood function, with a regularization term

$$
\begin{array}{r}
L=\log Z-\sum_{i, a} h_{i}(a) f_{i}(a)-\sum_{i<j, a, b} J_{i j}(a, b) f_{i j}(a, b)+ \\
\lambda_{J} \sum_{i<j, a, b}\left|J_{i j}(a, b)\right|^{2}+\lambda_{h} \sum_{i, a}\left|h_{i}(a)\right|^{2}
\end{array}
$$

where $Z=\sum_{s} \exp \left[\sum_{i} h_{i}\left(s_{i}\right)+\sum_{i<j} J_{i j}\left(s_{i}, s_{j}\right)\right]$ is the partition function, with $s$ running over the entire space of sequences.

For all other cases involving larger systems, we used the pseudo-likelihood maximization method plmDCA ${ }^{25,34}$ for approximate inference, with L2 regularization on both fields $\left(\lambda_{h}\right)$, and on couplings $\left(\lambda_{J}\right)$. The value of $\lambda_{h}$ is set consistent with past work to be $\lambda_{h}=0.01$ and the values of $\lambda_{J}$ as indicated in the main text.

For sequences of length $L$ and number of amino acids $q$, the couplings and correlations comprise four dimensional $L \times L \times q \times q$ arrays, and to represent them in as two-dimensional matrices, we take the Frobenius norm over amino acids, defined by

$$
\left\|X_{i j}\right\|=\left(\sum_{a, b} X_{i j}(a, b)^{2}\right)^{1 / 2}
$$

For couplings, this projection is gauge dependent and we implement it in the the zero-sum (or Ising) gauge, such that

$$
\sum_{a} J_{i j}(a, b)=\sum_{b} J_{i j}(a, b)=0, \quad \sum_{a} h_{i}(a)=0 .
$$

This gauge minimizes the Frobenius norm over all gauges. Note however that the inferred model $\hat{P}(s)$ is independent of the choice of the gauge. For comparison with input values $J$ inp, the inferred values $\hat{J}$ are represented as $\left\|\hat{J}_{i j}\right\| /\left\|J_{i j}^{\text {inp }}\right\|$ which is by definition 1 when the inference is correct.

\section{Multiple sequence alignment}

Sequences of the AroQ family were acquired by three rounds of PSI-BLAST ${ }^{35}$ using residues 1-95 of EcCM (the chorismate mutase (CM) domain of the E.coli CMprephenate dehrdratase) as the intial query (e-score cutoff $10^{-4}$ ). For alignment, we created a position-specific amino acid profile from $3 \mathrm{D}$ alignment of four $\mathrm{CM}$ atomic structures (PDB IDs 1ECM, 2D8E, 3NVT, and 1YBZ) and iteratively aligned nearest neighbor sequences from the PSI-BLAST using MUSCLE ${ }^{36}$, each time updating the profile. The resulting multiple alignment was subject to minor hand adjustment using standard rules and trimmed sequentially (1) to retain positions present in $\mathrm{EcCM},(2)$ to remove sequences with less than 82 positions, (3) to remove sequences with more than $30 \%$ gaps, and to remove excess sequences with more than $90 \%$ identity to each other. The final alignment contains 1259 sequences and 96 positions.

\section{Deep mutation library}

A saturation single site mutational library for $\mathrm{EcCM}$ was constructed using oligonucleotide-directed NNS codon mutagenesis. To mutate each position, two mutageneic oliognucleotides (one sense, one antisense) were synthesized (IDT) that contain sequences complementary to $\sim 15$ base pairs (bp) on either side of the target position and an NNS codon at the target site ( $\mathrm{N}$ is a mixture of $A, T, C, G$ bases and $S$ is a mixture of $G$ and $C$ ). One round of PCR was carried out with either the sense or antisense oligonucleotide and a flanking antisense or sense primer. A second round amplification with first round products and both flanking primers produced the full-length double-stranded product, which was purifed on agarose gel and quantitated using Picogreen (Invitrogen). All first round products were pooled in equimolar ratios, purified, digested with NdeI and XhoI, and ligated into correspondingly digested plasmid pKTCTET$0^{37}$. For selection, the library was transformed into electrocompetent NEB 10-beta cells (NEB) to yield i1000x transformants per gene, cultured overnight in $500 \mathrm{ml}$ LB supplemented with $100 \mu \mathrm{g} / \mathrm{ml}$ ampicillin (Amp), and subject to plasmid purification. The library was diluted to $1 \mathrm{ng} / \mathrm{ml}$ to minimize multiple transformation and transformed into the CM-deficient strain KA12 
bioRxiv preprint doi: https://doi.org/10.1101/2021.04.22.441025; this version posted April 23, 2021. The copyright holder for this preprint (which was not certified by peer review) is the author/funder, who has granted bioRxiv a license to display the preprint in perpetuity. It is made available under aCC-BY-NC-ND 4.0 International license.

Undersampling and the inference of coevolution in proteins $-10 / 12$

containing the auxilliary plasmid pKIMP-UAUC ${ }^{38}$ to yield $i 1000 x$ transformants per gene. The mixture was then recultured in $500 \mathrm{ml}$ LB containing $100 \mu \mathrm{g} / \mathrm{ml} \mathrm{Amp}$ and $30 \mu \mathrm{g} / \mathrm{ml}$ chloramphenicol (Cam) overnight, supplemented with $16 \%$ glycerol, and frozen at $-80^{\circ} \mathrm{C}$.

\section{Chorismate mutase selection assay}

The selection assay followed a recently reported proto$\mathrm{col}^{37}$. Briefly, glycerol stocks of KA12/pKIMP-UAUC carrying the saturation mutation library in pKTCTET-0 were cultured overnight at $30^{\circ} \mathrm{C}$ in LB supplemented with $100 \mu \mathrm{g} / \mathrm{ml}$ Amp and $30 \mu \mathrm{g} / \mathrm{ml}$ Cam. The culture was diluted to $O D_{600}$ of 0.045 in M9c minimal medium ${ }^{37}$ supplemented with $100 \mu \mathrm{g} / \mathrm{ml} \mathrm{Amp,} 30 \mu \mathrm{g} / \mathrm{ml} \mathrm{Cam}$, and $20 \mu \mathrm{g} / \mathrm{ml}$ each of L-phenylalanine (F) and L-tyrosine (Y) (M9cFY, non-selective conditions), grown at $30^{\circ} \mathrm{C}$ to $O D_{600} \sim 0.2$, and washed in M9c (no FY). An aliquot of the washed culture was used to innoculate $2 \mathrm{ml} \mathrm{LB}$ with $100 \mu \mathrm{g} / \mathrm{ml} \mathrm{Amp}$, and grown overnight at $37^{\circ} \mathrm{C}$ and harvested for plasmid purification (the pre-seleted, or input sample). For selection, another aliquot of the washed culture was diluted to a calculated starting $O D_{600}=$ $10^{-4}$ into $500 \mathrm{ml} \mathrm{M9c}$ supplemented with $100 \mu \mathrm{g} / \mathrm{ml}$ Amp, $30 \mu \mathrm{g} / \mathrm{ml} \mathrm{Cam}, 3 \mathrm{ng} / \mathrm{ml}$ doxycycline (to induce $\mathrm{CM}$ gene expression from the $P_{\text {tet }}$ promoter) and grown at $30^{\circ} \mathrm{C}$ for $24 \mathrm{~h}$ to a final $O D_{600}<0.1$. Fifty $\mathrm{ml}$ of the culture was harvested, resuspended in $2 \mathrm{ml}$ LB with 100 $\mu \mathrm{g} / \mathrm{ml}$ Amp, grown overnight at $37^{\circ} \mathrm{C}$, and harvested for plasmid purification (the seelcted sample).

Input and selected samples were amplified using two rounds of PCR with KOD polymerase (EMD Millpore) to add adapters and indicies for Illumina sequencing. Amplification in the first round included 6-9 random bases to aid initial focusing and part of the i5 or i7 Illumina adapters. The remaining adapter sequenes and TruSeq indicies were addred in the second round. PCR was limited to 16 cycles and included high initial tempate concentration to minimize amplification bias. Final products were gel purified (Zymo Research), quantified by Qubit (ThermoFisher), and sequenced on an Illumina MiSeq system with a paired-end 250 cycle kit. Paired-end reads were joined using FLASH, trimmed to the NdeI and XhoI cloning sites and translated. Only exact matches to library variants were counted. Relative enrichments (r.e.) were calculated according to the equation r.e. $=\log \left(f_{s}^{x} / f_{i}^{x}\right)-\log \left(f_{s}^{r} / f_{i}^{r}\right)$ where $f_{s}^{x}$ and $f_{i}^{x}$ represent the frequencies of each allele $x$ in either selected (s) or input $i$ pools and $f_{s}^{r}$ and $f_{i}^{r}$ represent those values for $\mathrm{EcCM}$, the wild-type reference.

\section{Acknowledgments}

We thank M. Weigt, R. Monasson, S. Cocco, F. Zamponi, A.F. Bitbol, Y. Meir, N.S. Wingreen and members of the Ranganathan and Rivoire laboratories for discussions. This work was supported by grant FRM
AJE20160635870 (O.R.), grant ANR 17-CE30-0021-02 (O.R.), NIH grant RO1GM12345 (R.R.), a Data Science Discovery Award from the University of Chicago (R.R.) and a collaboration grant from the France-Chicago Center (R.R. and O.R.).

\section{References}

${ }^{1}$ Debora S. Marks, Lucy J. Colwell, Robert Sheridan, Thomas A. Hopf, Andrea Pagnani, Riccardo Zecchina, and Chris Sander. Protein 3D Structure Computed from Evolutionary Sequence Variation. PLOS ONE, 6(12):e28766, December 2011. Publisher: Public Library of Science.

${ }^{2}$ Debora S. Marks, Thomas A. Hopf, and Chris Sander. Protein structure prediction from sequence variation. Nature Biotechnology, 30(11):1072-1080, November 2012. Number: 11 Publisher: Nature Publishing Group.

${ }^{3}$ Faruck Morcos, Andrea Pagnani, Bryan Lunt, Arianna Bertolino, Debora S. Marks, Chris Sander, Riccardo Zecchina, José N. Onuchic, Terence Hwa, and Martin Weigt. Direct-coupling analysis of residue coevolution captures native contacts across many protein families. Proceedings of the National Academy of Sciences, 108(49):E1293-E1301, December 2011. Publisher: National Academy of Sciences Section: PNAS Plus.

${ }^{4}$ Sergey Ovchinnikov, Hahnbeom Park, Neha Varghese, Po-Ssu Huang, Georgios A. Pavlopoulos, David E. Kim, Hetunandan Kamisetty, Nikos C. Kyrpides, and David Baker. Protein structure determination using metagenome sequence data. Science, 355(6322):294298, January 2017. Publisher: American Association for the Advancement of Science Section: Report.

${ }^{5}$ Anne-Florence Bitbol, Robert S. Dwyer, Lucy J. Colwell, and Ned S. Wingreen. Inferring interaction partners from protein sequences. Proceedings of the $\mathrm{Na}$ tional Academy of Sciences, 113(43):12180-12185, October 2016.

${ }^{6}$ Thomas Gueudré, Carlo Baldassi, Marco Zamparo, Martin Weigt, and Andrea Pagnani. Simultaneous identification of specifically interacting paralogs and interprotein contacts by direct coupling analysis. Proceedings of the National Academy of Sciences, 113(43):12186-12191, October 2016.

${ }^{7}$ Qian Cong, Ivan Anishchenko, Sergey Ovchinnikov, and David Baker. Protein interaction networks revealed by proteome coevolution. Science, 365(6449):185-189, July 2019. Publisher: American Association for the Advancement of Science Section: Report.

${ }^{8}$ Matteo Figliuzzi, Hervé Jacquier, Alexander Schug, Oliver Tenaillon, and Martin Weigt. Coevolutionary 
bioRxiv preprint doi: https://doi.org/10.1101/2021.04.22.441025; this version posted April 23, 2021. The copyright holder for this preprint (which was not certified by peer review) is the author/funder, who has granted bioRxiv a license to display the preprint in perpetuity. It is made available under aCC-BY-NC-ND 4.0 International license.

Undersampling and the inference of coevolution in proteins $-11 / 12$

Landscape Inference and the Context-Dependence of Mutations in Beta-Lactamase TEM-1. Molecular Biology and Evolution, 33(1):268-280, January 2016.

${ }^{9}$ Thomas A. Hopf, John B. Ingraham, Frank J. Poelwijk, Charlotta P. I. Schärfe, Michael Springer, Chris Sander, and Debora S. Marks. Mutation effects predicted from sequence co-variation. Nature Biotechnology, 35(2):128135, 2017.

${ }^{10}$ Victor H Salinas and Rama Ranganathan. Coevolution-based inference of amino acid interactions underlying protein function. eLife, 7:e34300, July 2018. Publisher: eLife Sciences Publications, Ltd.

${ }^{11}$ R. R. Cheng, O. Nordesjö, R. L. Hayes, H. Levine, S. C. Flores, J. N. Onuchic, and F. Morcos. Connecting the Sequence-Space of Bacterial Signaling Proteins to Phenotypes Using Coevolutionary Landscapes. Molecular Biology and Evolution, 33(12):3054-3064, 2016.

12 William P. Russ, Drew M. Lowery, Prashant Mishra, Michael B. Yaffe, and Rama Ranganathan. Naturallike function in artificial WW domains. Nature, 437(7058):579-583, September 2005.

${ }^{13}$ Michael Socolich, Steve W. Lockless, William P. Russ, Heather Lee, Kevin H. Gardner, and Rama Ranganathan. Evolutionary information for specifying a protein fold. Nature, 437(7058):512-518, September 2005.

${ }^{14}$ William P. Russ, Matteo Figliuzzi, Christian Stocker, Pierre Barrat-Charlaix, Michael Socolich, Peter Kast, Donald Hilvert, Remi Monasson, Simona Cocco, Martin Weigt, and Rama Ranganathan. An evolutionbased model for designing chorismate mutase enzymes. Science, 369(6502):440-445, July 2020. Publisher: American Association for the Advancement of Science Section: Report.

${ }^{15}$ Richard N. McLaughlin, Frank J. Poelwijk, Arjun Raman, Walraj S. Gosal, and Rama Ranganathan. The spatial architecture of protein function and adaptation. Nature, 491(7422):138-142, November 2012.

${ }^{16}$ Najeeb Halabi, Olivier Rivoire, Stanislas Leibler, and Rama Ranganathan. Protein sectors: evolutionary units of three-dimensional structure. Cell, 138(4):774786, August 2009.

${ }^{17}$ Kimberly A. Reynolds, Richard N. McLaughlin, and Rama Ranganathan. Hot spots for allosteric regulation on protein surfaces. Cell, 147(7):1564-1575, December 2011.

${ }^{18}$ Olivier Rivoire. Elements of Coevolution in Biological Sequences. Physical Review Letters, 110(17):178102, April 2013. Publisher: American Physical Society.

${ }^{19}$ Simona Cocco, Remi Monasson, and Martin Weigt. From Principal Component to Direct Coupling Analysis of Coevolution in Proteins: Low-Eigenvalue
Modes are Needed for Structure Prediction. PLOS Computational Biology, 9(8):e1003176, August 2013. Publisher: Public Library of Science.

${ }^{20}$ Simona Cocco, Christoph Feinauer, Matteo Figliuzzi, Rémi Monasson, and Martin Weigt. Inverse statistical physics of protein sequences: a key issues review. Reports on Progress in Physics, 81(3):032601, January 2018. Publisher: IOP Publishing.

${ }^{21}$ Olivier Rivoire, Kimberly A. Reynolds, and Rama Ranganathan. Evolution-Based Functional Decomposition of Proteins. PLoS Computational Biology, 12(6), June 2016.

${ }^{22}$ Hugo Jacquin, Amy Gilson, Eugene Shakhnovich, Simona Cocco, and Rémi Monasson. Benchmarking inverse statistical approaches for protein structure and design with exactly solvable models. PLoS computational biology, 12(5):e1004889, 2016.

${ }^{23}$ Olivier Rivoire. Parsimonious evolutionary scenario for the origin of allostery and coevolution patterns in proteins. Physical Review E, 100(3):032411, 2019.

${ }^{24}$ Barbara Bravi, Riccardo Ravasio, Carolina Brito, and Matthieu Wyart. Direct coupling analysis of epistasis in allosteric materials. PLoS computational biology, 16(3):e1007630, 2020.

${ }^{25}$ Magnus Ekeberg, Cecilia Lövkvist, Yueheng Lan, Martin Weigt, and Erik Aurell. Improved contact prediction in proteins: using pseudolikelihoods to infer potts models. Physical Review E, 87(1):012707, 2013.

${ }^{26}$ Matteo Figliuzzi, Pierre Barrat-Charlaix, and Martin Weigt. How pairwise coevolutionary models capture the collective residue variability in proteins? Molecular biology and evolution, 35(4):1018-1027, 2018.

${ }^{27}$ Stanley D Dunn, Lindi M Wahl, and Gregory B Gloor. Mutual information without the influence of phylogeny or entropy dramatically improves residue contact prediction. Bioinformatics, 24(3):333-340, 2008.

${ }^{28}$ Ivan Anishchenko, Sergey Ovchinnikov, Hetunandan Kamisetty, and David Baker. Origins of coevolution between residues distant in protein 3D structures. Proceedings of the National Academy of Sciences, 114(34):9122-9127, August 2017. Publisher: National Academy of Sciences Section: Biological Sciences.

${ }^{29}$ Chitra Narayanan, Donald Gagné, Kimberly A Reynolds, and Nicolas Doucet. Conserved amino acid networks modulate discrete functional properties in an enzyme superfamily. Scientific reports, 7(1):1-9, 2017.

${ }^{30}$ Allison S Walker, William P Russ, Rama Ranganathan, and Alanna Schepartz. Rna sectors and allosteric function within the ribosome. Proceedings of the National Academy of Sciences, 117(33):19879-19887, 2020. 
bioRxiv preprint doi: https://doi.org/10.1101/2021.04.22.441025; this version posted April 23, 2021. The copyright holder for this preprint (which was not certified by peer review) is the author/funder, who has granted bioRxiv a license to display the preprint in perpetuity. It is made available under aCC-BY-NC-ND 4.0 International license.

Undersampling and the inference of coevolution in proteins $-12 / 12$

${ }^{31}$ Gürol M. Süel, Steve W. Lockless, Mark A. Wall, and Rama Ranganathan. Evolutionarily conserved networks of residues mediate allosteric communication in proteins. Nature Structural Biology, 10(1):59-69, January 2003. Number: 1 Publisher: Nature Publishing Group.

${ }^{32}$ Marko Novinec, Matevž Korenč, Amedeo Caflisch, Rama Ranganathan, Brigita Lenarčič, and Antonio Baici. A novel allosteric mechanism in the cysteine peptidase cathepsin $\mathrm{k}$ discovered by computational methods. Nature communications, 5(1):1-10, 2014.

${ }^{33}$ Arjun S Raman, K Ian White, and Rama Ranganathan. Origins of allostery and evolvability in proteins: a case study. Cell, 166(2):468-480, 2016.

${ }^{34}$ Sivaraman Balakrishnan, Hetunandan Kamisetty, Jaime G Carbonell, Su-In Lee, and Christopher James Langmead. Learning generative models for protein fold families. Proteins: Structure, Function, and Bioinformatics, 79(4):1061-1078, 2011.

${ }^{35}$ Stephen F. Altschul, Thomas L. Madden, Alejandro A. Schäffer, Jinghui Zhang, Zheng Zhang, Webb Miller, and David J. Lipman. Gapped BLAST and PSIBLAST: a new generation of protein database search programs. Nucleic Acids Research, 25(17):3389-3402, 1997. _eprint: https://academic.oup.com/nar/articlepdf/25/17/3389/3639509/25-17-3389.pdf.

${ }^{36}$ Robert C. Edgar. MUSCLE: multiple sequence alignment with high accuracy and high throughput. Nucleic Acids Research, 32(5):1792-1797, 2004. eprint: https:/ / academic.oup.com/nar/articlepdf/32/5/1792/7055030/gkh340.pdf.

${ }^{37}$ Kathrin Roderer, Martin Neuenschwander, Giosiana Codoni, Severin Sasso, Marianne Gamper, and Peter Kast. Functional Mapping of Protein-Protein Interactions in an Enzyme Complex by Directed Evolution. PLOS ONE, 9(12):e116234, December 2014. Publisher: Public Library of Science.

${ }^{38}$ P. Kast, M. Asif-Ullah, N. Jiang, and D. Hilvert. Exploring the active site of chorismate mutase by combinatorial mutagenesis and selection: the importance of electrostatic catalysis. Proceedings of the National Academy of Sciences, 93(10):5043-5048, May 1996. Publisher: National Academy of Sciences Section: Research Article. 